\title{
Arthrocentesis: A Minimally Invasive Approach for Temporomandibular Joint Pain
}

\author{
${ }^{1}$ Snehal H Thakkar, ${ }^{2}$ Mukul Padhye, ${ }^{3}$ Yogesh Kini, ${ }^{4}$ Rujuta Desai, ${ }^{5}$ Vaibhav Shah, ${ }^{6}$ Suraj Pawar \\ ${ }^{7}$ Vruturaj Shevale, ${ }^{8}$ Frank Mehta
}

\begin{abstract}
The aim of this article is to provide emergency care to the diseased temporomandibular joint (TMJ) when conservative methods have failed to do so and to increase mouth opening, especially in cases of closed lock.

Our study was carried out on 18 healthy adult individuals who reported to our institute complaining of pain in one or both TMJs and restricted mouth opening. Mouth opening of each patient was recorded in $\mathrm{mm}$ prior to the procedure and pain recorded on a visual analog scale (VAS).

Arthrocentesis was performed using $100 \mathrm{cc}$ of Lactated Ringers solution on these individuals after conservative management of these individuals failed, followed by injection of Dexamethasone in the superior joint space. Pain was once again recorded on a VAS and mouth opening in $\mathrm{mm} 1$ day after the procedure, then after 1 week, 1,3 and 6 months.

In this sample, mean mouth opening (MMO) prior to the procedure was $24.3 \mathrm{~mm}(\mathrm{SD} \pm 3.114)$, ranging from 18 to $30 \mathrm{~mm}$.

MMO postarthrocentesis was $37.85 \mathrm{~mm}$ (SD \pm 7.457 ), ranging from 28 to $55 \mathrm{~mm}$.

From this study, the following conclusions may be drawn:

Arthrocentesis is an effective mode of treatment for closed lock of the TMJ when conservative methods have failed. Mouth opening is seen to increase in patients with restricted mouth opening due to closed lock of the TMJ. It provides instant relief from pain and has long lasting effects.
\end{abstract}

Keywords: Arthrocentesis, Temporomandibular joint, Pain, TMJ dysfunction, Joint lavage.

How to cite this article: Thakkar SH, Padhye M, Kini Y, Desai R, Shah V, Pawar S, Shevale V, Mehta F. Arthrocentesis: A Minimally Invasive Approach for Temporomandibular Joint Pain. Int J Head Neck Surg 2014;5(2):61-65.

Source of support: Nil

Conflict of interest: None

\section{INTRODUCTION}

Orofacial pain, including temporomandibular disorders (TMDs), is a common problem that, if misdiagnosed or

\footnotetext{
${ }^{1,6,7}$ Associate Professor, ${ }^{2}$ Professor and Head, ${ }^{3}$ Professor 4,5,8 Lecturer

${ }^{1-7}$ Department of Oral and Maxillofacial Surgery, Dr DY Patil Dental College and Hospital, Navi Mumbai, Maharashtra, India

${ }^{8}$ Department of Pediatric and Preventive Dentistry, Dr DY Patil Dental College and Hospital, Navi Mumbai, Maharashtra, India
}

Corresponding Author: Snehal H Thakkar, Associate Professor Department of Oral and Maxillofacial Surgery, Dr DY Patil Dental College and Hospital, Nerul, Navi Mumbai, Maharashtra, India Phone: 02227709590, e-mail: snehalthakkar82@gmail.com inappropriately treated, may lead to chronic pain and major personal crisis for the patient.

It occurs in approximately $10 \%$ of the population.

Several possible contributing factors, such as bruxism, postural habits, or emotional factors, may also complicate patient evaluation and, if neglected, can lead to inadequate or transient clinical outcomes.

If orofacial pain continues without resolution, emotional and psychosocial problems such as depression, anxiety, sleep disturbances, task avoidance, and lifestyle disturbances may occur and further complicate the problem.

A final diagnosis should be made based on the history, clinical examination, testing and imaging data. The Wilke's ${ }^{1}$ classification system of internal derangements of temporomandibular joint (TMJ) is helpful for classifying the severity of the underlying disease. Using this system to stage internal derangements also guides therapeutic choices and ultimately the choice of surgical technique.

Internal derangements usually involve partial or complete anteromedial disk displacement, which may be reducing or permanent. A permanently displaced disk probably restricts condylar translatory ability. With time the disk tends to deform and to become less mobile, relative to the articular eminence. This situation may predispose to the development of upper compartment adhesions. Mechanical compression and stretching of the retrodiscal tissues in a joint with a displaced disk is thought to be a frequent source of pain.

Nonsurgical treatment is the primary focus for patients experiencing symptoms and a limitation in function due to TMDs.

Oral and maxillofacial surgeons are continuously on the look-out for new treatment options for TMJ disorders, which cause minimum morbidity and at the same time provide satisfactory results.

Arthrocentesis is minimally invasive and according to most studies provides relief to most patients. It is an inexpensive procedure and can be carried out under local anesthesia on an out-patient basis. Hence, this procedure is now widely gaining acceptance.

\section{AIMS AND OBJECTIVES}

- To evaluate the effect of arthrocentesis in the management of closed lock of the TMJ. 
- To provide emergency care of the diseased TMJ when conservative methods have failed to:

1. Increase the mouth opening (MO).

2. Reduce the pain (recorded on a visual analog scale).

\section{MATERIALS AND METHODS}

The study was conducted in the Department of Oral and Maxillofacial Surgery at the Pad. Dr DY Patil Dental College and Hospital. Eighteen healthy, adult individuals (20 joints) complaining of pain on one or both sides of the TMJ on opening the mouth.

\section{CRITERIA FOR SELECTION OF PATIENTS}

- Healthy adult individuals were selected for this study.

- Patients complaining of pain in one or both TMJ.

- Patients with restricted mouth opening $(<30 \mathrm{~mm})$.

- Patients who had failed to respond to conservative therapy including restricting mouth opening, soft foods, local moist heat application and muscle relaxants.

- Patients whose preoperative MRI revealed anteriorly displaced disk.

- Patients who were available for regular follow-ups for 6 months following the procedure.

Patients were asked to fill a questionnaire prior to the procedure along with an informed consent for arthrocentesis and for carrying out the study. On clinical examination, maximum mouth opening and maximum painless mouth opening were recorded. Pain was recorded on a VAS of 1 to 10 prior to the procedure, 1 being no pain and 10 being maximum unbearable pain.

\section{TECHNIQUE}

The patient is seated at an angle of $45^{\circ}$, with the head turned to the unaffected side to provide an easy approach to affected side. The site is scrubbed with betadine solution and draped, the external auditory meatus blocked with cotton. A line is drawn from the middle of the tragus to the outer canthus. The posterior entrance point is located along the canthotragal line $10 \mathrm{~mm}$ from the middle of the tragus and $2 \mathrm{~mm}$ below it (point A). The anterior point of entry is placed $10 \mathrm{~mm}$ farther along the line and $10 \mathrm{~mm}$ below it (point $\mathrm{B}$ ). These markings indicate the location of the articular fossa and the eminence of the TMJ (Fig. 1).

Local anesthesia (LA) is injected at the planned entrance points avoiding penetration into the joint and injection into the synovial fluid. An 18 gauge needle connected to a $1 \mathrm{ml}$ syringe filled with Ringer's lactate (RL) is inserted into the superior compartment at the articular fossa aided by palpation. Two to $3 \mathrm{ml}$ of RL is injected to distend the upper point spaces.

The 2nd 18 gauge needle is then inserted into the distended compartment in the area of articular eminence to enable free flow of the RL through the superior compartment (Fig. 2).

$100 \mathrm{cc}$ of RL is injected to wash the joint space since this volume is sufficient to wash out denatured hemoglobin and various proteinases. During lavage, the mandible is moved through opening, excursive and protrusive movements to facilitate lyses of adhesions.

Lysis of adhesions is achieved by intermittent distension of the joint space by momentarily blocking the outflow needle and injecting under pressure during lavage.

At the end of the procedure, one needle is removed and $1 \mathrm{cc}$ dexamethasone is injected into the joint space since:

1. It is a potent, long acting corticosteroid, and

2. It has known anti-inflammatory properties.

Patient is recalled the next day for a check up, then after 1 week, 1,3 and 6 months and maximum mouth opening is recorded again. Pain once again is recorded on a VAS of 1 to 10 .

\section{RESULTS}

The study was conducted on 18 patients, twelve of which were female. Mean age of the patients was 38.8 years, ranging from 28 to 63 years of age ( $\mathrm{SD} \pm 9.684)$. In this sample, mean mouth opening (MMO) prior to the procedure was $24.3 \mathrm{~mm}(\mathrm{SD} \pm 3.114)$, ranging from 18 to $30 \mathrm{~mm}$.

Mean mouth opening postarthrocentesis was $37.85 \mathrm{~mm}$ ( $\mathrm{SD} \pm 7.457$ ), ranging from 28 to $55 \mathrm{~mm}$.

Decrease in pain on VAS was seen in all patients. Mean decrease on VAS- 3.55 units. Maximum decrease was 4 units and minimum decrease was 2 units (Tables 1 to 3 ).

\section{DISCUSSION}

Temporomandibular joint pain has plagued humankind for centuries and till date, we are on the look-out for the ideal

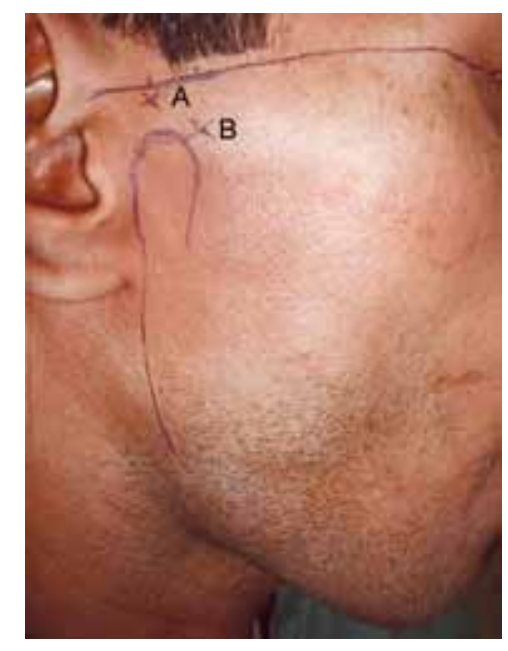

Fig. 1: Surface markings for insertion of needles. The first $X$ from the tragus indicates point $(A)$, the second $X$ lying anteriorly indicates point (B) 


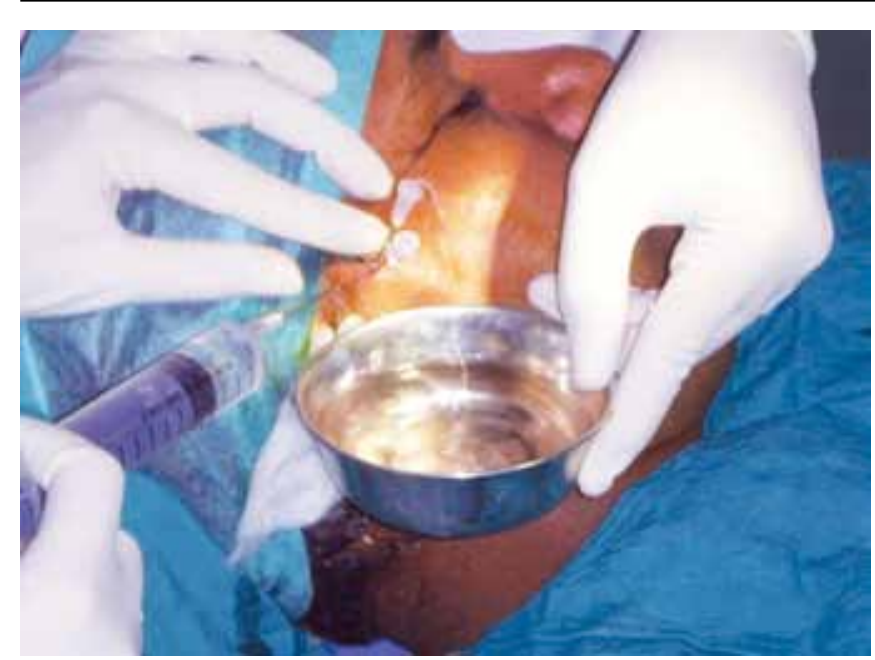

Fig. 2: The procedure being carried out under local anesthesia

treatment modality, which should be minimally invasive and at the same time, provide effective and long lasting results.

The treatment of TMJ pain lies in investigating and classifying the type of pain and this depends on the team of specialists which include the maxillofacial surgeon, the psychiatrist and the radiologist.

The aim of TMJ pain is to make an intolerable situation tolerable. Modalities used in the initial management of TMDs usually are nonsurgical. Should conservative methods fail, only then must one opt for surgery.

Controversy still surrounds the role of surgery in the management of pain and dysfunction of the TMJ, although only about $5 \%$ of all patients being treated got TMJ disorders are actually operated on.

Table 1: Pain on VAS prearthrocentesis

\begin{tabular}{ll}
\hline Vas & Frequency \\
\hline 5 & 3 \\
6 & 4 \\
7 & 7 \\
8 & 5 \\
9 & 1 \\
\hline Total & 20 \\
\hline
\end{tabular}

Table 2: Pain on VAS postarthrocentesis

\begin{tabular}{ll}
\hline Vas & Frequency \\
\hline 2 & 5 \\
3 & 8 \\
4 & 3 \\
5 & 4 \\
\hline Total & 20 \\
\hline
\end{tabular}

Arthrocentesis, being minimally invasive and providing desired results, is rapidly gaining acceptance as the treatment modality of choice for TMJ pain, if conservative methods fail.

The physical action of the lysis and lavage in the superior joint space rather than repositioning of the disk is thought to be responsible for the success of this procedure. It is thought to break down adhesions within the joint and remove inflammatory mediators including cytokines and interleukins, which result in chronic pain. Relief of TMJ pain also leads to improvement in both mouth opening and dysfunction.

Careful patient selection is important for this procedure. Exclusion criteria would include any previous invasive procedures on the TMJ, evidence of psychological problems (including diagnosis of atypical facial pain or clinically evident depression). These factors are similar to those used by $\mathrm{J}$ Kunjur et $\mathrm{al}^{2}$ in their study in 2003.

It has been proved that arthrocentesis is comparable to TMJ arthroscopy in relieving symptoms of closed lock according to the study published by JF Sanroman in 2003. ${ }^{3}$

A number of irrigating solutions have been used in varying quantities at different pressures.

Intraoperatively, the jaw has been manipulated to increase the mouth opening and, in addition, various medications have been instilled into the upper joint space post arthrocentesis, with varying results.

Arthrocentesis has been claimed to alter the viscosity of the synovial fluid, thereby, aiding translation of the disk and condyle. In addition, high pressure arthrocentesis in combination with shearing forces generated by joint manipulation is thought to release adhesions, thereby enabling increased mouth opening. The analgesic effect is claimed to be secondary to the washing out of inflammatory mediators and by direct action of the instilled medications on intracapsular pain receptors.

Our study was carried out at the Padmashree Dr DY Patil Dental College and Hospital in the Department of Oral and Maxillofacial Surgery. It was conducted on a sample of 18 patients and 20 TMJ. Of these, 12 were females and 6 males. The patients included in the study were in the age group of 28 to 63 years, the mean age being 38.90 years.

The preoperative mouth opening of the patients selected for the study was a maximum of $30 \mathrm{~mm}$ and pain on the VAS recorded as 5 or more.

Our technique was similar to that described by Nitzan et al. ${ }^{4}$ It involved insertion of two 18 -gauge needles into

Table 3: Mean and standard deviation for mouth opening pre and postarthrocentesis (in $\mathrm{mm}$ )

\begin{tabular}{lllll}
\hline Groups & $\begin{array}{l}\text { Mean mouth opening } \\
\text { (in mm) }\end{array}$ & Standard deviation & $\begin{array}{l}\text { Minimum mouth opening } \\
\text { (in mm) }\end{array}$ & $\begin{array}{l}\text { Maximum mouth opening } \\
\text { (in mm) }\end{array}$ \\
\hline Prearthrocentesis & 24.3 & 3.114 & 18 & 30 \\
Postarthrocentesis & 37.85 & 7.457 & 28 & 55 \\
\hline
\end{tabular}


the superior joint space of the TMJ under LA. Through one needle, we injected $100 \mathrm{ml}$ of lactated Ringer's solution into the superior joint space. Kaneyama et $\mathrm{al}^{5}$ suggested that 300 to $400 \mathrm{ml}$ solution be used for the washout of bradykinin, IL-6 and proteins. We however found $100 \mathrm{ml}$ to be sufficient to relieve the patient's symptoms.

The second needle acted as an outflow portal, which allows lavage of the joint cavity. Lysis of adhesions is achieved by intermittent distension of the joint space by momentarily blocking the outflow needle and injecting under pressure during lavage. At the end of the procedure, the exit portal was removed. The superior needle is not removed. Dexamethasone $(2 \mathrm{mg}$ in $1 \mathrm{ml}$ ) was injected through the portal of entry at the end of the lavage to alleviate intracapsular inflammation. We based our success on subjective as well as objective results. We defined success a satisfactory increase in mouth opening as well as satisfactory decrease in pain on the VAS.

Merrill $^{6}$ added sclerotherapy to arthroscopic lysis, lavage, and manipulation by injecting $1 \mathrm{ml}$ of a 1 to $3 \%$ solution of sodium tetradecyl sulphate into the posterior disk space. He reported a 91 to $96 \%$ success rate which was consistent with the $95 \%$ success rate in our study which included injection of $1 \mathrm{ml}$ of dexamethasone into the upper joint space.

In our study, mean prearthrocentesis mouth opening was $24 \pm 3.114 \mathrm{~mm}$ and 6 months postarthrocentesis, mean mouth opening was $37.85 \pm 7.457 \mathrm{~mm}$. The mean increase in mouth opening was thus $13.55 \mathrm{~mm}$.

One-hundred percent of our patients had a satisfactory increase in the mouth opening.

In 2001, GH Alpaslan and C Alpaslan ${ }^{7}$ published a study to compare the efficacy of TMJ arthrocentesis with and without injection of sodium hyaluronate in treatment of internal derangements. The mean increase in mouth opening in the first group (arthrocentesis with injection of sodium hyaluronate) was $9.54 \mathrm{~mm}$ and that in the second group (arthrocentesis only) was $7.22 \mathrm{~mm}$. The results achieved by US at the end of 6 months were superior to this study (mean increase in mouth opening being $13.55 \mathrm{~mm}$ ).

In 1995, G Dimitroulis, MF Dolwick, A Martinez et al ${ }^{8}$ published a follow-up study on TMJ arthrocentesis and lavage for the treatment of closed lock. A course of physiotherapy is commenced immediately postoperatively along with nonsurgical treatment for TMDs such as medication and in some patients', occlusal splint therapy. These postoperative physiotherapy, medication and splints were not used by us in our study since it is impossible to assess which of the treatments were responsible for the success. The study undoubtedly shows an increase in mouth opening of $17.7 \mathrm{~mm}$ as compared to $13.55 \mathrm{~mm}$ increase in mouth opening at the end of our study, but due the above-mentioned reasons, there is no way to prove the increased mouth opening is solely due to arthrocentesis.

In our study, the mean pain on VAS prearthrocentesis was 6.85 and postarthrocentesis, was 3.3.

In one patient, the pain score on the VAS reduced from 8 to 5 . He however, complained of persistent pain and discomfort on opening the mouth. This is the only case we have considered as a failure.

In the study conducted by Alpaslan and Alpaslan in $2001,{ }^{7}$ the mean pain on the VAS in the first group (arthrocentesis with sodium hyaluronate injection) reduced from 5.5 to 0 . In the second group, mean pain on the VAS reduced from 7.5 to 2 . In our study, mean pain on the VAS reduced from 6.85 to 3.3 . The subjective results thus achieved in the study with injection of sodium hyaluronate are superior to those achieved by us by injection of dexamethasone.

In the study published by G Dimitroulis et $\mathrm{al}^{8}$ in 1995 , the pain was recorded on the VAS preoperatively and postoperatively as in our study but the VAS is normally accepted as a scale of 1-10. The authors have deviated from this scale by increasing the length of the scale, this makes comparison with other studies difficult.

Outcome analysis of major studies on arthrocentesis by Segami et $\mathrm{al}^{9}$ (1990), Murakami et $\mathrm{al}^{10}$ (1987), and Nitzan et $\mathrm{al}^{11}$ (1991) pertaining to the treatment of TMJ closed lock have shown success rates between 70 and 100\% which are consistent with the findings of our study which had a $95 \%$ success rate.

The only complication we faced was swelling of the neighboring tissues due to perfusion of Ringer's solution, which was consistent with the findings of Dorrit Nitzan ${ }^{12}$ and G Dimitroulis et al. ${ }^{8}$ This too, was transient and lasted for a maximum of 3 hours in all patients.

Potential complications, although not recorded in this study have been documented in literature. These are as follows:

- Temporary facial paresis or paralysis caused by the use of a local anesthetic. However, these effects are transient and disappear within a few hours. ${ }^{12}$

- Joint contamination. ${ }^{13}$

- Local irritation caused by introduction of foreign materials. ${ }^{12}$

- Hematoma with potential for infection. ${ }^{12}$

- Numerous other complications associated with arthrocentesis have been described. A 59-year-old woman remained drowsy and developed left hemiparesis after TMJ arthrocentesis, caused by an extradural hematoma. ${ }^{14}$ Our study highlights the advantages of arthrocentesis. They are as follows:

- It is a minimally invasive procedure and is therefore preferred to surgical intervention.

- It can be carried out under local anesthesia on an out patient basis.

- It is an inexpensive procedure. 
- It has minimum incidence of failure when properly performed.

- It provides instant relief of pain and increase in mouth opening hence prolonged recovery period is eliminated.

- If results are not satisfactory, the procedure can be repeated.

- Risk of associated complications is minimal. The complication encountered by us, that is, swelling of surrounding tissues due to extravasation of fluid, was transient and lasted only 2 to 3 hours.

The disadvantages of this procedure are as follows:

- It is extremely technique and operator sensitive.

- The surface markings described in the technique are not reliable and must be aided with palpation of the condylar head to ensure entry into the superior joint space.

- There is no way to confirm the entry of the needle into the superior joint space.

- Since, the needles are not fixed in position, changes in direction of the needles changed the rate of outflow of the Ringer's lactate and sometimes, were also dislodged.

- Since, the lavage is carried out in the superior compartment of the joint, the inferior joint space is not involved in the procedure. Hence, any pathology existing in this compartment is not addressed by the arthrocentesis thereby giving us less than ideal results.

\section{CONCLUSION}

From this study, the following conclusions may be drawn: Arthrocentesis is an effective mode of treatment for closed lock of the TMJ when conservative methods have failed. Mouth opening is seen to increase in patients with restricted mouth opening due to closed lock of the TMJ. It provides instant relief from pain and has long lasting effects.

\section{REFERENCES}

1. Wilkes $\mathrm{CH}$. Internal derangements of the temporomandibular joint. Pathological variations. Arch Otolaryngol Head Neck Surg 1989;115(4):496-497.
2. Kunjur J, Anand R, Brennan PA, et al. An audit of 405 TMJ arthrocentesis with intra-articular morphine infusion. Br J Oral Maxillofac Surg 2003;41(1):29-31.

3. Sanroman JF. Closed lock (MRI fixed disc): A comparison of arthrocentesis and arthroscopy. Int J Oral Maxillofac Surg 2004; 33(4):344-348.

4. Nitzan DW, Dolwick MF, Martinez GA. Temporomandibular joint arthrocentesis: a simplified treatment for severe, limited mouth opening. J Oral Maxillofac Surg 1991;49(11):1163-1167.

5. Kaneyama K, Segami N, Nishimura M, et al. The ideal lavage volume of for removing bradykinin, interleukin06, and protein from the temporomandibular arthrocentesis. J Oral Maxillofac Surg 2004;62(6):657-661.

6. Merrill RG. Arthroscopic temporomandibular joint lysis, lavage, and manipulation and chemical sclerotherapy for painful hypermobility and recurrent mandibular dislocation. In: Clark G, Sanders B, Bertolami C, editors. Advances in diagnostic and surgical arthroscopy of the temporomandibular joint. Philadelphia: WB Saunders;1993. p. 75-84.

7. Alpaslan GH, Alpaslan C. Efficacy of TMJ arthrocentesis with and without injection of sodium hyaluronate in treatment of internal derangement. J Oral Maxillofac Surg 2001;59(6): 613-618.

8. Dimitroulis G, Dolwick MF, Martinez A. Temporomandibular joint arthrocentesis and lavage for the treatment of closed lock: a follow-up study. Br J Oral Maxillofac Surg 1995;33(1):23-27.

9. Segami N, Murakami KI, Iizuki T. Arthrographic evaluation of disc position following mandibular manipulation technique for internal derangement with closed lock of the temporomandibular joint. J Craniomandib Disord 1990;4(2):99-108.

10. Murakami KI, Iizuki T, Matsuki M, et al. Recapturing the persistent anterior displaced disc by mandibular manipulation after pumping and hydraulic pressure to the upper upper joint cavity to the temporomandibular joint. Cranio 1987;5(1):17-24.

11. Nitzan DW, Dolwick MF, Martinez GA. Temporomandibular joint arthrocentesis: a simplified treatment for severe, limited mouth opening. J Oral Maxillofac Surg 1991;49(11):1163-1167.

12. Nitzan DW. Arthrocentesis-incentives for using this minimally invasive approach for temporomandibular disorders. Oral Maxillofac Surg Clin North Am 2006 Aug;18(3):311-328.

13. Ostensson A, Geborek P. Septic arthritis as a nonsurgical complication in rheumatoid arthritis: relation to disease severity and therapy. Br J Rheumatol 1991;30(1):35-38.

14. Carroll TA, Smith K, Jakubowski J. Extradural haematoma following temporomandibular joint arthrocentesis and lavage. Br J Neurosurg 2000;14(2):1524. 